\title{
50 years of artificial intelligence: A neuronal approach
}

\section{Introduction}

Recently, the 50th anniversary of the birth of Artificial Intelligence (AI) has been celebrated worldwide [1-4], and about 65 years ago (1943) its foundational works on Biocybernetics and Bionics were published due to movements led by McCulloch and Pitts [5] and Wiener [6]. Since then, a part of the scientific community has been interested in looking to Biology from Physics, Mathematics, Engineering and Computation with a double proposal. On the one hand, there has been search for inspiration on new concepts and mechanisms of interest in Engineering and Computation giving rise to Bionics in first place, and currently to the "bio-inspired" movement. On the other hand, the concepts and techniques proper of Physics, Engineering and Calculus are used to help neuroscientists to understand the functioning of biological systems, giving rise to Biocybernetics first and more recently to Computational Neuroscience, which includes Neurodynamics for the neuronal level, and to the techniques of representation and knowledge usage, proper of AI, for the cognitive level.

This "50 Years of Artificial Intelligence: Campus Multidisciplinary in Perception and Intelligence" special issue collects a subset of the best papers in the fields of Neurocomputing, presented at the Campus Multidisciplinary in Perception and Intelligence, CMPI-2006 [1]. The CMPI-2006 international conference, held in Albacete, Spain, during July 10-14, 2006, resulted in a forum for scientists in commemoration of the 50th Anniversary of Artificial Intelligence, which successfully reported recent research advances of $\mathrm{AI}$ and where the participants exchanged knowledge in close relation to the state-of-theart of Perception and Intelligence.

We want to highlight the multidisciplinar character of the CMPI-2006 encounter. Multidisciplinarity is a nonintegrative mixture of disciplines in which each discipline retains its methodologies and assumptions without change or development from other disciplines within the multidisciplinary relationship. Precisely, neural computing is a multidisciplinary field that attracts psychologists, physicists, computer scientists, neuroscientists, and AI investigators, among others. The success of the conference is directly related to the excellent results of AI research in Spain and other Spanish speaking countries. In this special issue revised and extended versions of works previously presented at CMPI-2006 and essentially related to the sensorial functions (of the visual and auditory pathway) and the tasks of character recognition and selective visual attention (SVA) are included.

\section{Special issue composition}

This special issue starts with two papers that make a historical sweeping of the 50 years of Artificial Intelligence from two different points of view. In "Symbols versus connections: Fifty years of Artificial Intelligence" J. Mira reviews the history of AI from its original stage to the current paradigms dominated by connectionism and symbolic approaches. Some causes of the disparity between the initial optimistic expectations and the modest results obtained are analyzed. In this work, the author attempts: (1) to approach the concept of AI both as a science of the natural and as knowledge engineering (KE), (2) to summarize some of the conceptual, formal and methodological approaches to the development of AI during the last 50 years, (3) to mention some of the constitutive differences between human knowing and machine knowing and (4) propose some suggestions that we believe must be adopted to progress in developing AI. And, in "On the nature of neural information: A critique of the received view 50 years later" Barandiaran and Moreno offer a critical review of the concept of neural information, as received within mainstream neuroscience from AI. This conception of information is constructed as a conditional probability of a stimulus given a certain neural activation, a correlation that cannot be accessed by the organism and fails to explain its causal organization. In this paper, the authors reconstruct an alternative conception of neural information: a pattern of signals that is selected by the organism (as an autonomous system) to contribute to its self-maintenance by virtue of its correlation with external conditions, a correlation that might further be evaluated by the very system.

The next two papers introduce computational models for some functionality proper of the auditory and visual systems, respectively. "Rate versus time representation of highfrequency spectral notches in the peripheral auditory system: A computational modeling study" by E.A. Lopez-Poveda, 
A. Alves-Pinto, A.R. Palmer and A. Eustaquio-Martín introduces a computational model of the peripheral auditory system, which is used to explain the paradoxical observation that discriminating between broadband noise sounds with and without high-frequency spectral notches is more difficult at mid-intensities than at lower or higher intensities. The simulations suggest that the discrimination task in study relies on comparing the timing of auditory nerve spikes, and hence that high-frequency sounds are represented in the auditory nerve by a time code. The authors further suggest that the improvement in spectral discrimination at high intensities is associated with inherent inner hair cell non-linearities. The other article, " $A$ conceptual frame with two neural mechanisms to model selective visual attention processes", written by J. Mira, A.E. Delgado, M.T. López, A. Fernández-Caballero and M.A. Fernández, proposes a re-writing in terms of knowledge models of the double bottom-up/top-down architecture used in order to theorize and to experiment in the attentional processes. The conjecture is that there are two basic mechanisms (lateral inhibition and accumulative computation) for the synthesis of a great part of the SVA processes. In this work the authors explore a way of saving the semantic gap between the analytic formulations of the neuronal models and the concepts of the natural language used to describe the cognitive processes for the case of the attentional processes, consisting in (1) proposing in first place a conceptual model of the attention double bottom-up/top-down organization, (2) proposing afterwards a neurophysiological model of the cortical and sub-cortical involved structures, (3) establishing the correspondences between the entities of (1) and (2), (4) operationalizing the model by using biologically inspired calculation mechanisms (algorithmic lateral inhibition and accumulative computation) formulated at symbolic level, and, (5) assessing the validity of the proposal by accommodating the works of the research team to diverse aspects of attention associated to visual surveillance tasks.

The next three papers introduce some neurally inspired applications. Firstly, "Topos: Spiking Neural Networks for Temporal Pattern Recognition in Complex Real Sounds" by P. González-Nalda and B. Cases depicts the approach used to build the Topos application, a simulation of twowheeled robots able to discern real complex sounds. Topos is framed in the nouvelle concept of subsymbolic AI, applied to the field of evolutionary robotics. This paper focuses on the simulation of biologically inspired artificial cochleas and spiking neural networks, in order to model the embodied control system of the robots. The method chosen to find the most appropriate parameters that determine robots' behaviour is evolutionary computation techniques, with the aim of avoiding any human intervention in this task. Then, "ANN+GIS: An automated system for property valuation" by N. García Rubio, M. Gámez Martínez and E. Alfaro Cortés states that, although property valuation models have become an important paradigm in real-estate market research, the results of the most well-known approaches are limited due to various data-related problems such as the non-linearity of relationships, the presence of noise, and the absence of necessary information. The authors aim to overcome these obstacles. Their paper introduces an automated system for property valuation that combines artificial neural network models with a geographic information system. The artificial neural network models used in this work are the multilayer perceptron, the radial basis function, and Kohonen's maps. A third application paper is called "A Robust Model of the Neuronal Regulator of the Lower Urinary Tract Based on Artificial Neural Networks", by F. Maciá Pérez, J.M. García Chamizo, A. Soriano Payá and D. Ruiz Fernández. The authors propose a new system for the neuronal regulator of the lower urinary tract from a model based in a multi-agent system in which each neuronal centre corresponds with an agent. This system incorporates a heuristic in order to make it more robust in the presence of possible inconsistencies. The heuristic used is based on a neural network (Orthogonal Associative Memory). Knowledge through training has been added to the system, using correct patterns of behaviour of the urinary tract and behaviour patterns resulting from dysfunctions in two neuronal centres as a minimum.

The last three papers are devoted to enhancing previous results in neural computing techniques. Thus, in "On the application of different evolutionary algorithms to the alignment problem in statistical machine translation", L. Rodríguez, I. García-Varea and J.A. Gámez propose several evolutionary algorithms for computing alignments between two sentences in a parallel corpus in statistical machine translation. Alignments are used, on the one hand, to train the statistical models and, on the other, during the decoding process to link the words in the source sentence to the words in the partial hypotheses generated. In both cases the quality of the alignments is crucial for the success of the translation process. In "A retinomorphic architecture based on discrete-time cellular neural networks using reconuurable computing", J.J. Martínez, F.J. Toledo, E. Fernández and J.M. Ferrández describe a novel architecture for the hardware implementation of non-linear multi-layer cellular neural networks (CNNs). This makes it feasible to design CNNs with millions of neurons accommodated in low-price FPGA devices, being able to process standard video in real time. This architecture has been used to build a CNN-based model of the synapsis I of the fovea region, with the aim of implementing the basic spatial processing of the retina in reconfigurable hardware. The model is based on the receptive fields of the bipolar cells and mimics the retinal architecture achieving its processing capabilities. Lastly, "Parametric improvement of lateral interaction in accumulative computation in motionbased segmentation" by J. Martínez-Cantos, E. Carmona, A. Fernández-Caballero and M.T. López deals with segmentation of moving objects. In this paper an artificial neuronal network approach for moving object 
segmentation which uses accumulative computation and recurrent lateral interaction, called LIAC, is revisited. The aim of the authors is to improve segmentation provided by LIAC in a double sense: by removing the detected objects not matching some size or compactness constraints and by learning suitable parameters that improve the segmentation behaviour through a genetic algorithm.

All these papers are representative of the current state of knowledge and show the situation of some of its frontiers, such as the distinction among signal, information and knowledge, the neuronal coding, and the problems in character recognition.

\section{Acknowledgements}

We would like to thank all the authors for their highquality contributions, and the reviewers for agreeing to perform the reviews.

\section{References}

[1] Campus Multidisciplinary in Perception and Intelligence. Available at $\langle$ http://www.info-ab.uclm.es/cmpi/ $\rangle$.

[2] The Dartmouth Artificial Intelligence Conference: The Next 50 Years. Available at $\langle$ http://www.dartmouth.edu/ ai50/homepage.html $\rangle$.

[3] The Twenty-First National Conference on Artificial Intelligence. Available at $\langle$ http://www.aaai.org/Conferences/AAAI/aaai06.php $\rangle$.
[4] The 50th Anniversary Summit of Artificial Intelligence. Available at $\langle$ http://www.isi.imi.i.u-tokyo.ac.jp/\%7Emaxl/Events/ASAI50MV/ $\rangle$.

[5] W. McCulloch, W. Pitts, A logical calculus of the ideas immanent in nervous activity, Bull. Math. Biophys. 5 (1943) 115-133.

[6] N. Wiener, Cybernetics, or Control and Communication in the Animal and the Machine, Wiley, Inc, New York, 1948.

Antonio Fernández-Caballero Departamento de Sistemas Informáticos, Instituto de Investigación en Informática de Albacete (I3A), Universidad de Castilla-La Mancha, Escuela Politécnica Superior de Albacete, 02071 Albacete, Spain E-mail address: caballer@dsi.uclm.es

José Mira Departamento de Inteligencia Artificial, Escuela Técnica Superior de Ingeniería Informática, Universidad Nacional de Educación a Distancia, 28040 Madrid, Spain

Gustavo Deco Department of Technology, Computational Neuroscience, Institució Catalana de Recerca i Estudis Avancats (ICREA), Universitat Pompeu Fabra, 08003 Barcelona, Spain 